\title{
CASO CLIINICO - VIDEOFORUM
}

\section{SONDAS DE NUTRICIÓN CON APOYO ENDOSCOPICO}

\section{NUTRITION TUBES WITH ENDOSCOPIC SUPPORT}

\section{Hervás Molina AJ, Serrano Ruiz FJ}

UGC Aparato Digestivo. Hospital Universitario Reina Sofía. Córdoba.

La necesidad de colocación de sondas de nutrición es cada vez más frecuente. El acceso nutricional, cuando el paciente tiene alguna patología que le impide o dificulta la ingesta, podemos realizarlo con diferentes tipos de sondas, nasogástricas, nasoenterales, de una o dos luces, mediante gastrostomía por el sistema push o pull, gastrostomía con extensión yeyunal. Para su colocación podemos utilizar diferentes tipos de endoscopios, convencionales o ultrafinos para uso transnasal, con o sin apoyo radiológico.

La nutrición por sonda está indicada en aquellos pacientes que tienen una incapacidad para la deglución, ya sea de causa neurológica o por alteración anatómica tras una cirugía ORL o maxilofacial; presencia de estenosis del tramo alto del tubo digestivo, orgánica o funcional; fístulas posquirúrgicas o por enfermedad inflamatoria intestinal; en pancreatitis graves; trastornos de la evacuación gástrica; necesidad de suplementar a la nutrición oral como en estados de desnutrición grave de causa

\section{CORRESPONDENCIA}

Antonio José Hervás Molina

Hospital Universitario Reina Sofía

14004 Córdoba

ahervasm@live.com

Fecha de envio: 26/06/2021

Fecha de aceptación: 10/08/2021 psiquiátrica, oncológica o malabsortiva; o estados hipercatabólicos. Las contraindicaciones son la necesidad de una cirugía urgente - la presencia de una hemorragia digestiva alta aguda.

La elección del tipo de sonda la haremos según la localización de estenosis que queramos superar o el punto al que queramos llevar la alimentación según el estado de la función gástrica y el riesgo de broncoaspiración. Disponemos de sondas de diferentes longitudes, calibres, así como sondas de luz simple o luz doble. Las sondas de doble luz son de utilidad cuando existe una alteración de la evacuación gástrica y la alimentación debe ser realizada a nivel distal y además es necesaria una aspiración del contenido gástrico. No todas las sondas están preparadas para su utilización con guía, por lo que deberemos comprobar este aspecto, aunque en muchas será suficiente con cortar el extremo distal para que la guía pueda pasar. También deberemos asegurar que dispone de orificios laterales y si no los tuviera, realizar unos ojales laterales con la tijera.

Antes de comenzar el procedimiento deberemos hacer una planificación según las necesidades del paciente, elegir y tener a disposición todo el material necesario (Tabla 1). Es importante confirmar que la guía que hemos elegido desliza adecuadamente por el interior de la sonda. Como ayuda utilizaremos agua, lubricante hidrosoluble o aceite de oliva. Algunas guías con cubierta teflonada no deslizan correctamente por lo que deberemos probar diferentes modelos hasta encontrar la más adecuada. 
La disponibilidad de un endoscopio ultrafino nos facilitará la inserción de la sonda ya que al utilizar un acceso transnasal no será necesaria la reconversión de la guía de oral a nasal. En el caso de no disponer de este modelo de endoscopio, la reconversión de la guía de oral a nasal la haremos con la ayuda de una sonda de aspiración que introduciremos por nariz y la pasaremos a boca con la ayuda de nuestros dedos. En estos casos, la guía no deberá ser demasiado rígida para que pueda ser manipulada sin producir daño al paciente.

\section{Tabla 1. Material que puede ser necesario.}

\section{Sonda de nutrición enteral}

- Elegir diámetro y longitud

- Luz central distal o con opción de cortar el extremo distal

- Naso-gástrica o naso-enteral

- Habitual $12 \mathrm{~F}$ y $110 \mathrm{~cm}$

- Otras opciones: $10 \mathrm{~F}$ y $140 \mathrm{~cm}$

- Doble luz

- Vía nasal $16 \mathrm{~F}$ o $18 \mathrm{~F}$ luz gástrica / $9 \mathrm{~F}$ luz enteral

- Vía gastrostomía: sonda balón de 18 F o 20 F con extensión yeyunal

Guía

- Probar diversos modelos para asegurar que desliza adecuadamente

- Probar flexibilidad si necesidad de reconversión boca-nariz

- Valorar guías de balones de dilatación TTS, que no han sido

utilizadas, para uso con endoscopio ultrafino

Sistema de introducción por válvula del canal de trabajo

- Cono de inserción de las guías de CPRE

Lubricante

- Lubricar endoscopio

- Lubricar guía

Jeringa con agua

- Lubricación de la sonda

- Comprobación permeabilidad de la sonda

Endoscopio

- Gastroscopio ultrafino

- 4-5 mm. (diámetro inserción)

- Vía transnasal y vía gastrostomía

- Gastroscopio diagnóstico

- $\quad$ 9-10 mm. (diámetro inserción)

$$
\text { - Vía oral }
$$

\section{Fluoroscopia}

- Necesaria para

- Estenosis neoplásicas o complejas, que precisen dilatación

- Naso-enterales

- Gastro-enterales

- Doble luz

- No disponibilidad de endoscopio ultrafino: Vía oral

\section{Clips}

- Necesidad de arrastrar la sonda en paralelo con el endoscopio

- Sonda sin luz central distal

Sistema de reconversión boca-nariz

- Si no disponibilidad endoscopio ultrafino

- Sonda de aspiración recortada

\section{Sistema de fijación nasal}

- Esparadrapo

- Sistema de retención nasal Corgrip $®$ (opcional)

Riesgo de extracción

- Recolocación tras extracción accidental
En algunas ocasiones tendremos que avanzar la sonda en paralelo con el endoscopio. Algunas sondas están diseñadas con un saliente o con un hilo en forma de anillo, que puede ser atrapado con una pinza. Si no lo tuviera podremos realizarlo con un hilo de sutura anudado a través de pequeños orificios en el extremo distal de la sonda y con forma de anillo. La experiencia indica que es más cómodo atrapar este extremo con un clip, ya que en ocasiones la pinza no consigue soltarse de la sonda y sin embargo el clip podemos liberarlo con facilidad, quedando suelto de su introductor.

El control radiológico no siempre es necesario, sobre todo en sondas cuyo extremo va a quedar en estómago o no es necesaria una posición duodenal distal. Sin embargo, cuando se indica una sonda en estenosis antrales, pilóricas o duodenales, cuando el extremo distal debe quedar en duodeno distal o yeyuno proximal o en las sondas de doble luz, el control radiológico durante su colocación es de gran ayuda ya que nos permite confirmar la adecuada progresión de la sonda y realizar las correcciones que sean necesarias, evitando recolocaciones.

La fijación de la sonda a la nariz se suele hacer con un espadrapo que podemos cortar o bien es suministrado con la sonda. Este sistema es frágil, por lo que la sonda puede salirse de forma inadvertida o con un pequeño tirón. En los pacientes en los que deseemos una forma de fijación más segura podemos utilizar un sistema denominado Corgrip ${ }^{\circledR}$, que consiste en pasar un hilo por detrás del tabique nasal, quedando sus extremos saliendo por ambas fosas nasales y que mediante un clip de cierre quedarán fijados a la sonda.

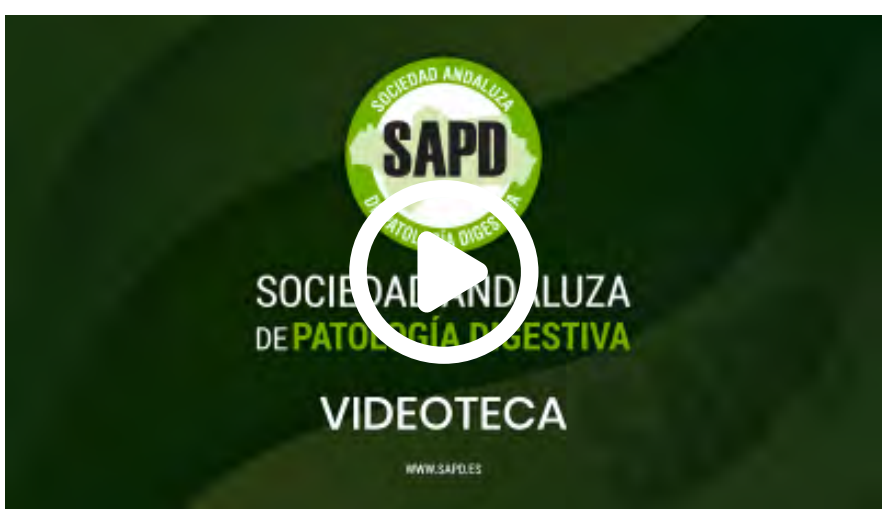

Acceder al vídeo

\section{Bibliografía}

1. Cerezo Ruiz A, Naranjo Rodríguez A, Hervás Molina AJ, et al. Utilidad de la endoscopia ultrafina transnasal en la aplicación de sondas de nutrición enteral. Gastroenterol Hepatol 2008;31:631-4.

2. Shastri YM, Shirodkar M, Mallath MK. Endoscopic feeding tube placement in patients with cancer: a prospective clinical audit of 2055 procedures in 1866 patients. Aliment Pharmacol Ther. 2008 27:649-58.

3. Zhang L, Huang YH, Yao W, et al. Transnasal esophagogastroduodenoscopy for placement of nasoenteric feeding tubes in patients with severe upper gastrointestinal diseases. J Dig Dis. 2012;13:310-5. 